\title{
Recording of relaxation characteristics in molding of polyvinylchloride
}

\author{
Ekaterina $V$. Solovyova ${ }^{1, *}$ \\ ${ }^{1}$ Vologda state university, Architectural department
}

\begin{abstract}
The article presents the basic physical and chemical characteristics of polymers affecting the molding process of products for various purposes, the relaxation curves of polyvinyl chloride in a wide range of temperatures and strains.
\end{abstract}

\section{Introduction}

Volumetric products for generally technical and engineering purpose made of polyvinyl chloride (PVC) have been widely used in various fields of the national economy: the automotive industry, aircraft, electrical engineering, etc. Vacuum molding is used in the technological process of plastics processing, which provides high operational strength properties and increases technical characteristics of such volumetric products.

The basis of plastic processing processes are physical and physical-chemical processes of structure formation and molding:

- heating, melting, glass-transition and cooling;

- volume and size changes in case of exposure of temperature and pressure;

- deformation accompanied by the development of plastic (irreversible) and highly elastic deformation and orientation of macromolecular chains;

- relaxation processes;

- formation of supramolecular structure, crystallization of polymers (crystallizing);

- destruction of polymers.

These processes can take place simultaneously and inter connected. During the molding process, the polymer heated by a high temperature, deformation by shear, tension or compression and then cooled. Depending on the parameters of these processes, it is possible significantly change the structure, conformation of macromolecules, as well as physical-mechanical, optical and other characteristics of polymers.

\section{Methods}

At a certain stage of vacuum forming the most responsible are the relaxation processes occurring in the polymer. This occurs when the cooling of the products proceeds slowly and without pressure, for example, when receiving products by extrusion on worm presses. In this case, there may be a change in the size of the product, the so-called "swelling" of the product, may appear roughness and even bumpiness on the surface of the products, change the shape of the product (elastic turbulence). Swelling of products occurs under the action of normal stresses, the action of which is perpendicular to the direction of flow of the jet. Normal stresses arise due to the tendency of oriented molecules to go into a non-stressed state (Wiesenberger effect).

Surface defects and distortion of the product shape are the result of relaxation of uneven stresses arising from unstable flow, which is not a consequence of flow transformation from laminar to turbulent. Even in conditions far from the turbulent flow in the stream of possible non-uniform strain, which is the consequence of shear deformation. Distortion of the form occurs at significant (critical) shear stresses, when the melt flow slips in certain areas of the forming tool, while the flow becomes irregular, which leads to occurrence of significant inhomogeneous stresses [1].

Thus, it is very important to know the relaxation parameters of a particular polymer in order to obtain the technological parameters of molding plastic products

\section{Results}

This article presents the relaxation curves of polyvinyl chloride obtained at a wide range of temperatures and strains. The tests carried out in the laboratory of polymer materials of INEOS RAS named after A. N. Nesmeyanov, head of the laboratory Professor A. A. Askadsky.

Figure 1 shows PVC stress relaxation curves defined at constant strain at different temperatures. The graph shows that even a small increase in temperature from 20 to $300 \mathrm{C}$ is accompanied by a sharp decline in the relaxing voltage, but with a further increase in temperature, the relaxation curves fit into a narrow beam. Only at a temperature of 500C approaching the softening temperature, stress relaxation occurs intensively in the region of long durations of the process.

* Corresponding author: evsvologda@mail.ru 
Calculated values of relaxation processes parameters [2] presented in table 1 . The parameter $\beta$, associated with the reaction order $\mathrm{n}$ of the interaction of relaxation oscillator, remains approximately the same at all temperatures; the kinetic constant of the interaction of relaxation oscillator increases with temperature. The correlation coefficient $r$ pending to 1 , and it is always higher when using the $\mathrm{T} 1(\tau)$ core than the T2 $(\tau)$ core. The An parameter, associated with the number of nonuniformity in the material has no definite dependence. The value of the initial stress $\sigma 0$, determined by calculation, changes little with increasing temperature, and the value of the quasi-equal stress $\sigma \infty$, naturally decreases with temperature increase. The characteristics of the initial $\sigma 0$ and quasi-equal $\sigma \infty$ are the most important characteristics of the relaxation process; they determine the performance of the polymer material.

Figure 2 shows the curves of stress relaxation of PVC in a wide interval of deformation. Such curves make it possible to identify the deformation region in which linear and nonlinear mechanical behavior observed.

As it shown on the figure that the stress relaxation curves naturally shift to the region of high stresses with the growth of deformation, and such a shift occurs smoothly. Modules go sharply down. Thus, nonlinear mechanical behavior starts with very small deformations.

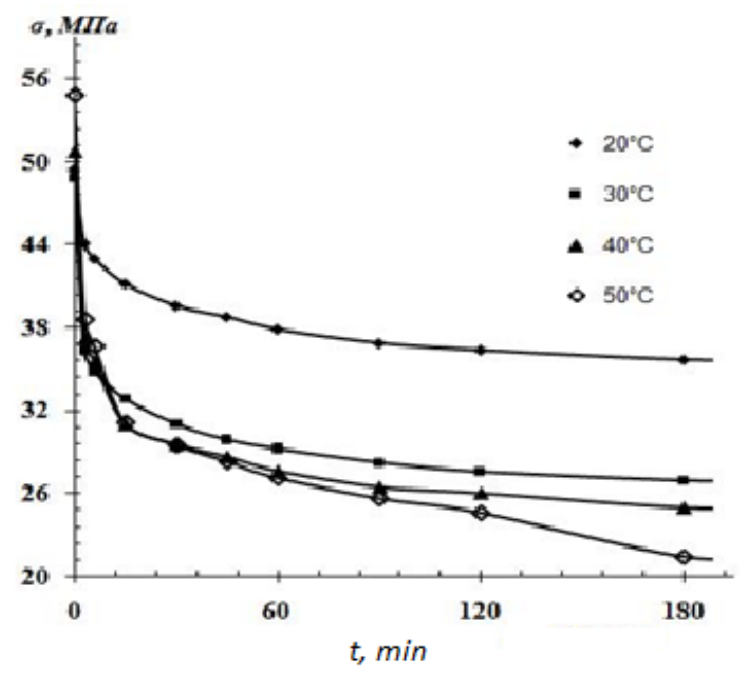

Fig. 1. PVC stress relaxation curves at different temperatures.

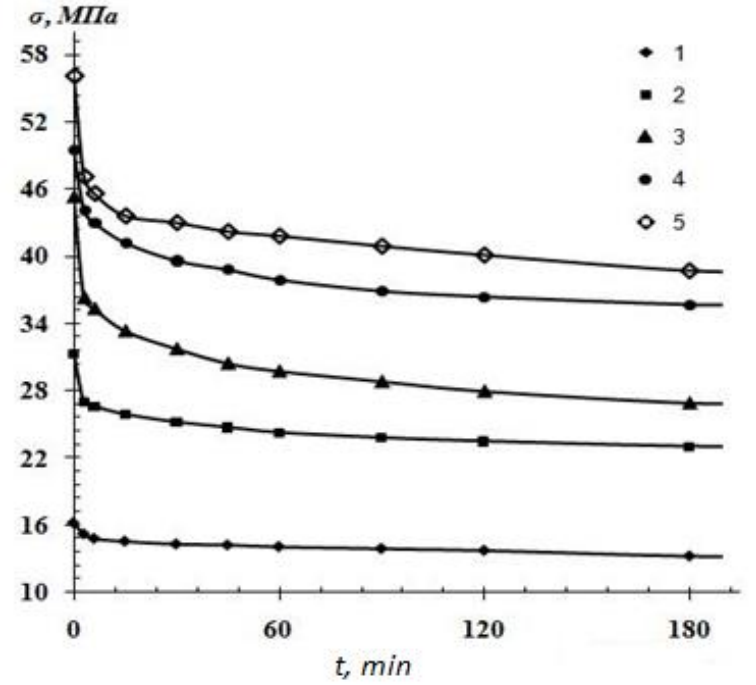

Fig. 2. PVC stress relaxation curves at room temperature determined at different strains (1) $-1 \%,(2)-2 \%,(3)-2,5 \%$, (4) $-3 \%,(5)-3,5 \%$.

Further, the stress relaxation curves were rearranged as a function of the relaxing module from time (Fig. 3). It can be seen that at deformation of more than $1 \%$ the curves of the relaxing module go sharply down. Thus, nonlinear mechanical behavior is observed starting with very small deformations.

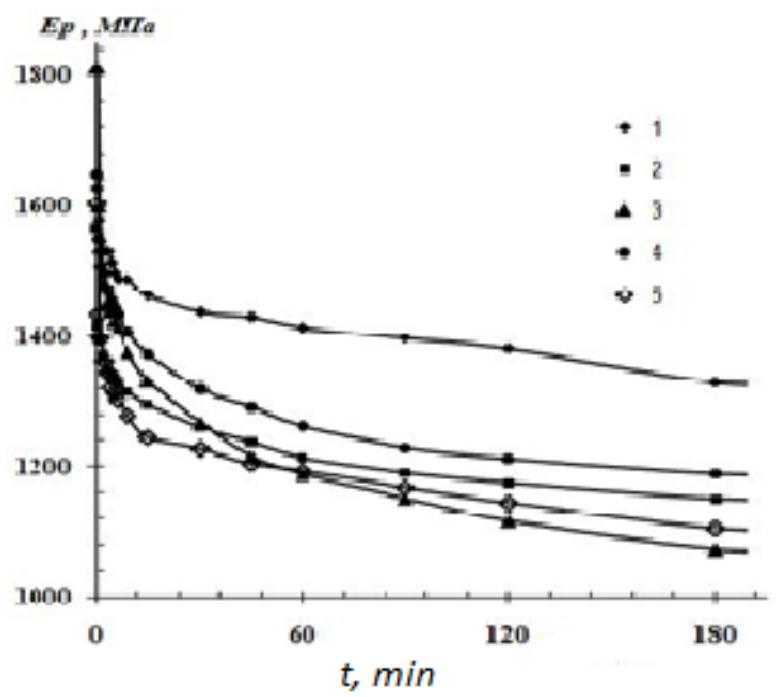

Fig. 3. Correspondence of a relaxing primary PVC module from time, determinated by different deformations (1) $-1 \%$, (2) $-2 \%,(3)-2,5 \%,(4)-3 \%,(5)-3,5 \%$.

Two types of stress relaxation curve calculations were performed. According to the first variant, all the stress relaxation curves of the primary PVC were calculated using the Boltzmann-Volterra equation (1).

$$
\sigma=\sigma_{0}\left[1-\int_{0}^{t} T(\tau) d \tau\right]
$$

where $\sigma$ - the relaxing stress, $\sigma 0$-the initial stress that develops at the end of the "instantaneous" deformation 


$$
\begin{gathered}
T_{1}(\tau)=-\frac{S_{0}}{k_{b} m_{1}}\left\{\frac{1}{\left[\frac{1}{(1+k \tau / \beta)^{\beta}}-\alpha_{0}\right] \ln \left[\frac{1}{(1+k \tau / \beta)^{\beta}}-\alpha_{0}\right]+\left[1-\frac{1}{(1+k \tau / \beta)^{\beta}}+\alpha_{0}\right] \ln \left[1-\frac{1}{(1+k \tau / \beta)^{\beta}}+\alpha_{0}\right]}-\frac{1}{\ln 0.5}\right\} \\
T_{2}(\tau)=-\frac{S_{0}}{k_{B} m_{2}}\left[\frac{1}{a \tau^{\gamma} \ln a \tau^{\gamma}+\left(1-a \tau^{\gamma}\right) \ln \left(1-a \tau^{\gamma}\right)}-\frac{1}{\ln 0,5}\right]
\end{gathered}
$$

task, T $(\tau)$ - the relaxation core, $\tau$-the current time, which runs values from 0 to $t$, $t$-the final time.

The cores $\mathrm{T} 1(\tau)$ and $\mathrm{T} 2(\tau)(2,3)$ were used [3].

The calculation results are given in table 1 . It can be seen that in all cases the correlation coefficient $r$ when using T1 $(\tau)$ is higher than the kernel T2 $(\tau)$, and approaches 1 . The value $\beta$, associated with the order of the reaction of the interaction of relaxation oscillators, for all deformations is 0.2 , i.e., there is a high order of interaction $\mathrm{n}$. The interaction kinetic constant does not have a clear dependence on the value of deformation, as well as the value An, characterizing the number of nonhomogeneities in the material.

\section{Discussion}

For PVC, according to the studies and the relaxation curves presented above, the nonlinearity of the mechanical behavior occurs, starting with a very small deformation of $2 \%$ (Fig. 3). The results of approximation of the relaxation behavior in the nonlinear domain are shown in figure 4 (for deformation of $2 \%$ ). Since the curve obtained at $2 \%$ strain deviates from the curve 1 obtained at $1 \%$ strain by more than $5 \%$, the first curve was adopted as the averaged curve, the core parameters T1 $(\tau)$ are shown in table 2. It should be noted, xnj for such an "averaged" curve, the correlation coefficient $r$ is 0.991 . For the curve obtained at $2 \%$ deformation, the correlation coefficient is 0.999 , and the value $\delta$ is 40

\begin{tabular}{|c|c|c|c|c|}
\hline Temperature & $20^{\circ} \mathrm{C}$ & $30^{\circ} \mathrm{C}$ & $40^{\circ} \mathrm{C}$ & $50^{\circ} \mathrm{C}$ \\
\hline \multicolumn{5}{|c|}{ Core $T_{1}(\tau)$} \\
\hline 1 & 2 & 3 & 4 & 5 \\
\hline$\beta$ & 0.3 & 0.3 & 0.2 & 0.3 \\
\hline 1 & 2 & 3 & 4 & 5 \\
\hline $\mathrm{k}$ & 0.001 & 0.01 & 0.01 & 0.01 \\
\hline $\mathrm{r}$ & 0.9969 & 0.9814 & 0.9871 & 0.9909 \\
\hline$A_{H}$ & $1.163 \cdot 10^{25}$ & $1.101 \cdot 10^{24}$ & $1.306 \cdot 10^{24}$ & $1.446 \cdot 10^{25}$ \\
\hline$\sigma_{\mathrm{o}}$ & 58.33 & 61.95 & 59.86 & 66.09 \\
\hline$\sigma_{\infty}$ & 31.91 & 27.31 & 23.72 & 15.04 \\
\hline \multicolumn{5}{|c|}{ Core $T_{2}(\tau)$} \\
\hline $\mathrm{a}$ & 0.0306 & 0.05 & 0.05 & 0.0306 \\
\hline$\gamma$ & 0.5 & 0.5 & 0.5 & 0.5 \\
\hline $\mathrm{r}$ & 0.9924 & 0.9504 & 0.9775 & 0.9778 \\
\hline A & $8.098 \cdot 10^{23}$ & $2.605 \cdot 10^{23}$ & $2.503 \cdot 10^{23}$ & $6.263 \cdot 10^{23}$ \\
\hline$\sigma_{\mathrm{o}}$ & 46.49 & 42.87 & 42.84 & 43.19 \\
\hline$\sigma_{\infty}$ & 35.60 & 28.04 & 26.48 & 22.22 \\
\hline \multicolumn{5}{|c|}{ Experimental value } \\
\hline$\sigma_{\text {нач }}$ & 49.40 & 48.82 & 50.76 & 54.76 \\
\hline$\sigma_{\text {кон }}$ & 35.70 & 26.96 & 24.97 & 21.37 \\
\hline
\end{tabular}

Table 1. Results of approximation of PVC stress relaxation curves, obtained at different temperatures, deformation 3\%. 
$\mathrm{cm} 3 / \mathrm{mol}$ (66.4 $\AA 3$ repeating link) (recall that the value $\delta$ is an excess free volume in which the elementary act of the relaxation process is played out). If we assume that this cavity of excess free volume has a spherical shape, then its size is $\sim 5,0 \AA 3$.

The next stage of the calculation was to compare the relaxation curves obtained at deformations of 1,2 and $2.5 \%$ (Fig. 4). The results showed (table. 2) that although the relaxation curve obtained at $2.5 \%$ deformation is in the nonlinear domain of mechanical behavior, the excess free volume $\delta=0$. In this case, the correlation coefficient $r=0.997$, i.e. close to 1 .

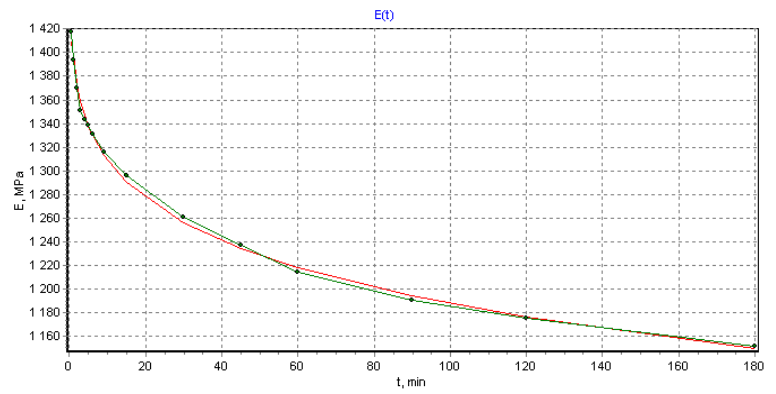

Fig. 4. Relaxation modulus curves for primary PVC at $2 \%$ deformation (points correspond to the experiment, the curve without points is calculated).

Table 2. The parameters of approximation of relaxation curves in PVC in the nonlinear domain of mechanical behavior.

\begin{tabular}{|c|c|c|}
\hline \multirow{2}{*}{$\mathbf{\varepsilon} 0 \%$} & \multicolumn{2}{|c|}{ Parameters } \\
\cline { 2 - 3 } & $\boldsymbol{r}$ & $\boldsymbol{\delta}, \mathbf{~ s m} \mathbf{3} / \mathbf{m o l}$ \\
\hline 1.0 & 0.999 & 0 \\
\hline 2.0 & 0.998 & 40.0 \\
\hline 2.5 & 0.997 & 0 \\
\hline 3.0 & 0.998 & 380.7 \\
\hline 3.5 & 0.992 & 0 \\
\hline
\end{tabular}

\section{Summary}

In conclusion, it should be told that relaxation are of great practical importance in the processing of polymers, and the processes occurring in this case can be understood and explained only using the theory of relaxation phenomena [1-3].

Practical conclusions can be drawn as follows: for the rapid development of orientation of macromolecules in the process of obtaining fibers and PVC films, it is necessary to increase the shear stress during molding and reduce the cooling time of the melt, so that the process of disorientation does not occur. When forming products by extrusion and injection molding, if it is necessary to reduce the anisotropy of shrinkage and mechanical strength, it is desirable to reduce the viscosity of the melt by increasing the temperature or using polymers with a lower molecular weight. Conversely, when a high anisotropy is needed, the relaxation time should be large, which is achieved by lowering the melt temperature and increasing the viscosity or by rapidly cooling the products.

\section{References}

[1] V.M. Sutyagin, A.A. Lyapov,General chemistry of polymer technology, Textbook, Tomsk: Publishing house of Tomsk Polytechnic University, 195 (2007).

[2] A.A. Askadsky, M.N. Popova, O.V. Pakhneva et al., Analysis of stress relaxation in the nonlinear region of mechanical stress, High Molecular compounds, 51, 5, 838-844 (2009).

[3] A.A. Askadsky,M.N. Popova, E.V. Solovyova, Research of relaxation properties of primary and secondary polyvinyl chloride, Intersectoral scientific and technical journal: Constructions from composite materials, Moscow: VIMY, 4 (2010). 